\title{
Computational Investigations of Cognitive Impairment in Huntington's Disease
}

\author{
Eddy J. Davelaar \\ Birkbeck College, University of London \\ United Kingdom
}

\section{Introduction}

Huntington's Disease (HD) is a genetic disorder involving progressive loss of the neostriatal cells. The most prominent symptom in early clinical HD is chorea, which is assumed to reflect a dysfunctional error-feedback system. Cognitive impairments are also observable in early stages of the disease in mild form at first, but more severe in later stages. Cognitive decline is present years before the clinical manifestation of HD. In a recent report, the term "mild cognitive impairment" in preclinical HD (pHD) was advocated with an amnestic and nonamnestic variant (Duff, et al., 2010). Considerable effort is invested in identifying which combinations of neuropsychological tests have high clinical utility.

Currently, there is no treatment for HD and much research addresses possible interventions, (see for extensive review, Zuccato, et al., 2010). In this chapter, the focus is on the profile of cognitive impairment during the period before clinical symptoms are present and is investigated via computational methods. A computational model of the basal ganglia is used to simulate cognitive performance. The cognitive tasks that have been shown to be sensitive to HD pathology cover such domains as memory (e.g., verbal learning), executive functioning (e.g., random sequence generation), and attention (e.g., flanker task). The HD pathology is simulated in the model and the pattern of cognitive decline is compared with published reports, when present.

This chapter is structured as follows. Section 2 reviews the functional neuroanatomy of the basal ganglia and summarizes the status of computational modeling of the basal ganglia. In section 3, the neuropathology and cognitive impairments in HD are reviewed and a new classification scheme of cognitive tasks is presented. To preview the scheme, tasks cluster in accordance with their reliance on the internal dynamics of the basal ganglia. A computational study will be presented in section 4 that addresses compensatory mechanisms in HD as well as specific deficits that differentiate early pHD from late pHD and amnestic pHD from nonamnestic pHD. The model sheds light on why certain tasks detect deficits at clinical stages (memory span), while other tasks detects deficits both at preclinical and clinical stages (episodic memory). The chapter concludes with a reflection on the utility of computational models in HD research. 


\section{The basal ganglia}

The basal ganglia are a group of functionally related subcortical nuclei that have predominantly been described as being involved in movement control. In the last decades, it has been shown that the basal ganglia are involved in many cognitive domains, such as learning, memory, and planning. The functionality of the basal ganglia can be understood by the interconnections among the nuclei and the various neurotransmitters used by the structures (for a recent update see, Delong \& Wichmann, 2009). Computational studies at various levels of biological realism have enhanced our understanding of the complex neurodynamics of the basal ganglia and its role in cognitive performance.

\subsection{Neuroanatomy of the basal ganglia}

The basal ganglia consist of the putamen, the caudate nucleus, the globus pallidus, the substantia nigra, and the subthalamic nucleus (see Fig. 1). The caudate nucleus and the putamen constitute the striatum (STR) and receive cortical input. The striatum projects to the globus pallidus and substantia nigra. The globus pallidus is divided into the internal (GPi) and external (GPe) segments. The substantia nigra also contains functionally separate parts: the pars compacta $(\mathrm{SNc})$ and the reticulata $(\mathrm{SNr})$. The GPi and the $\mathrm{SNr}$ project to nuclei in the thalamus and form the output of the basal ganglia. The thalamus is reciprocally connected with frontal cortical areas. This cascade of projections, i.e., cortex $\rightarrow$ BG $\rightarrow$ Thal $\rightarrow$ cortex, is referred to as the cortico-basal ganglia loop of which there are several, each with its specific functional role. The loops are thought to implement a selection mechanism through which only the most appropriate actions (or thoughts) are selected (Doya, 2007).
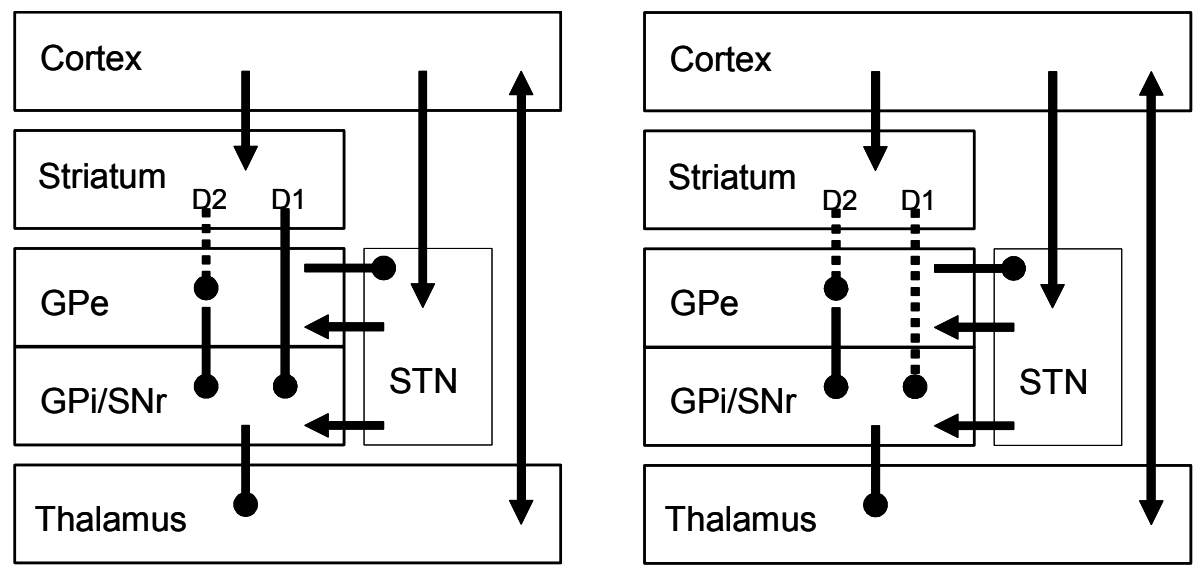

Fig. 1. Architecture of the basal ganglia. Not all connections are presented. Left: simplified situation in pHD with the degraded D2-pathway. Right: simplified situation in HD with both D1 and D2 pathways degraded.

There are several pathways within the basal ganglia in which the cortex projects to the basal ganglia and receive, via the thalamus, the output of the basal ganglia (see Fig. 1). In the direct pathway, projections go from the cortex to STR, then to GPi/SNr and then the thalamus. In the indirect pathway, projections go from the cortex to STR, then to GPe, then 
to the subthalamic nucleus (STN), then to GPi/SNr and then to the thalamus. A third pathway called the hyperdirect pathway (Nambu, et al., 2000, 2002) involves direct projections from the neocortex to the STN, which then influences the activation in the $\mathrm{GPi} / \mathrm{SNr}$.

The projections from the cortex to the striatum are topographically organized both in the anterior-posterior and in the lateral-medial directions (e.g., Nambu, 2011). This even extends to the level of specific body parts, such as the face, arm, and leg (Alexander \& Crutcher, 1990). Although this finding is consistent with a view that the various cortico-basal ganglia loops are separate parallel circuits, there is evidence showing that the loops share information (Graybiel, 1995) and that the amount of information sharing is modulated by dopamine (Bergman, et al., 1998).

The neurochemistry of the basal ganglia is complex with inhibitory and excitatory neurotransmitters and receptors distributed in a precise architecture. The striatum consists mainly of medium spiny neurons (MSN) that are predominantly GABA-ergic and contain either dopamine D1- or D2-receptor. MSNs with both D1- and D2-receptors have also been found (see for a review, Perreault, et al., 2011). The neural space of the striatum is made up of a large area called the matrix that is rich in acetylcholinesterase and smaller islands of acetylcholinesterase-poor neurons called striosomes (Cichetti, et al., 2000). Striosomal MSNs have a different input/output connectivity and receptor expression than matrix MSNs (Eblen \& Graybiel, 1995; Joyce, et al., 1986; Lévesque \& Parent, 2005), which has been interpreted to show a functional differentiation between the two compartments (see for review, Cichetti, et al., 2000).

The prevailing view is that the MSNs in the striosomes receive input from parts of the limbic system and project directly or indirectly to dopaminergic cells in the SNc (but see, Lévesque \& Parent, 2005). The MSNs in the matrix that contain D1-receptors project to the GPe/SNr using the neurotransmitters GABA and substance P and form the striatal-pallidal leg of the direct pathway. The GPe/SNr-neurons inhibit the thalamus and are tonically active. This makes the direct pathway one that disinhibits the reciprocal cortico-thalamic loop. It is important to stress that disinhibition is different from excitation in that excitation leads to thalamic activation in the absence of cortico-thalamic input, whereas disinhibition essentially lowers the threshold to allow already present cortico-thalamic input to activate the thalamic neurons (Chevalier \& Deniau, 1990). The MSNs in the matrix that express D2receptors contain GABA and enkephalin and project to the GPi, forming the striatal-pallidal leg of the indirect pathway. The GPe inhibits the STN, which has glutamatergic projections to GPe and GPi/SNr. The indirect and hyperdirect pathways thus increase the GPi/SNr activity, leading to more inhibition of the thalamus. Functionally, these pathways are preventing thalamic activation. The nigrostriatal dopaminergic projections increase the firing rate of MSNs of the direct pathway that contain D1-receptors and decrease the firing rate of MSNs of the indirect pathway that contain D2-receptors.

The striatal neurons are surrounded by various tonically active interneurons. The afferent and efferent connections of the interneurons respect the striosomal-matrix boundaries, apart from those of the cholinergic interneurons, which are located around the striosomal-matrix boundaries and are thought to be critical in allowing crosstalk between MSNs in both striatal areas (Cichetti, et al., 2000). 
The number of participating neurons decreases from cortex to striatum to globus pallidus. This convergence was interpreted as evidence that the basal ganglia play a role in evaluating contextual information for generating appropriate motor responses (Graybiel, 1991; Houk \& Wise, 1995; Joel \& Weiner, 1994). A complementary view is that the convergence of information together with the inhibitory interconnections is the neurobiological equivalent of dimensionality reduction through principal component analysis (Bar-Gad, et al., 2003). This process allows the cortex to focus on and learn the underlying statistical structure of a large amount of activity patterns.

\subsection{Computational models of the basal ganglia}

The connections among the basal ganglia structures form complex feedback loops that modulate each other at different time-scales. This continuous interaction makes it very hard to intuit how a single manipulation will affect the cortico-subcortical dynamics. This is even further complicated by the different timecourses of neurodegeneration of the two pathways in HD. Building and testing computational models will help in understanding the functional and impaired dynamics of the basal ganglia.

Many detailed computational models of the basal ganglia exist (for reviews on cognitive models see e.g., Bullock, 2004; Bullock, et al., 2009; Cohen \& Frank, 2009). Most models are concerned with the motor deficits seen in diseases such as Parkinson's Disease, but some have been developed to further understand the functional roles of the connections among subcortical nuclei (e.g., Gurney, et al., 2001) or the roles of the entire collection of basal ganglia in cognitive performance.

Computational models that investigate the functional roles of the basal ganglia vary in the level of biological realism of the neurons and in the level of detail regarding the interconnections among the basal ganglia. Models may employ (1) simplified rate-coding neurons that are used as a proxy for groups of individual firing neurons (e.g., Berns \& Sejnowski, 1998; Frank \& Claus, 2006; Frank, et al., 2001; Gurney, et al., 2001; Monchi, et al., 2000) or (2) spiking neurons with various levels of intracellular detail (e.g., J. Brown, et al., 1999; Guthrie, et al., 2009; Humphries, et al., 2006).

Many of these models address the role of the basal ganglia in specific cognitive domains, such as working memory (e.g., Berns \& Sejnowski, 1998; Frank, et al., 2001; Monchi, et al., 2000), decision making and action selection (e.g., Frank \& Claus, 2006; Gurney, et al., 2001; Guthrie, et al., 2009), and reinforcement learning (e.g., J. Brown, et al., 1999; Frank \& Claus, 2006). Although there is a bias towards addressing dopaminergic influences, which explains the large volume of computational models that address cognitive performance in Parkinson's Disease and schizophrenia, associative learning of cortico-striatal connections will not be addressed in this chapter. An extensive literature exists on the role of dopamine in reward-based or reinforcement learning and is closely related to the mechanisms of longterm potentiation and long-term depression (for recent review see, Manninen et al., 2010).

The various computational models that deal with cognitive phenomena are based on the original idea of the direct and indirect pathways (Albin, et al., 1989). However, recent modeling work has focused on the role of the STN (Frank, 2006; Gurney, et al., 2001). In particular, the hyperdirect pathway (Nambu, et al., 2000, 2002) has been attributed the function of relaying stopping decisions from the cortex (Frank, 2006). Cortical activation of 
the STN causes a global increase in GPe activation, which in turn prevents any channel from disinhibiting the thalamus. Gurney et al., like Nambu, et al. (2000, 2002), view the STN as an input structure complementing the striatum. The dual-input design creates an offcenter/on-surround in GPe, which leads to the selection of the relevant channel while inhibiting closely related channels. In both scenarios, the STN plays a more central role than in previous models (e.g., Albin, et al., 1989).

\section{Huntington's Disease}

Huntington's Disease is an autosomal-dominant progressive neurodegenerative disorder that presents with motor disturbances, psychiatric symptoms, and cognitive decline. The onset of clinical symptoms is in middle-age, but the disorder can manifest at any time between infancy and old age. In early HD, hyperkinesia is observed, whereas in later stages, hypokinesia dominates.

\subsection{Neuropathology of Huntington's Disease}

Huntington's Disease results from a gene mutation on chromosome 4, leading to an increase in CAG repeats. The gene codes for the protein huntingtin (htt) and the mutant variant thus has an extended number of glutamine repeats, varying from 11 to 34 in normal individuals and 36 to 121 units in HD patients (The Huntington's Disease Collaborative Research Group, 1993). The role of huntingtin is yet unclear, but evidence suggests that it is involved in neurodevelopmental processes (e.g., Hebb, et al., 2004). The pathophysiological mechanisms of Huntington's Disease are poorly understood, but research with transgenic animal models of the disorder is providing insights into the causative factors and potential treatments.

\subsubsection{Huntington's Disease involves targeted cell death}

The disease triggers striatal neurons to go into apoptosis (commit suicide) and the neuropathology spreads in all directions. In the early stage of the disease, there is up to $60 \%$ loss of GABA/enkephalin striatopallidal neurons that project to GPe. These neurons are part of the indirect pathway and express D2-receptors. The amount of D2-receptor binding sites correlates with the estimated years of disease onset (Feigin, et al., 2007). In the intermediate stage, up to $50 \%$ of $\mathrm{GABA} /$ substance $\mathrm{P}$ striatonigral neurons that project to $\mathrm{SNr}$ are lost. These neurons are part of the direct pathway and express D1-receptors. Finally, at the last stage, there is loss of GABA/substance P striatopallidal neurons that project to GPi that are also part of the D1-receptor expressing direct pathway (Glass, et al., 2000).

Animal studies with knock-in mice have verified the two-stage neurodegenerative process that starts with hyperkinesia and continues with hypokinesia, mirroring the progression of cell death from predominantly D2-receptor MSNs associated with the indirect pathway followed by the D1-receptor MSNs that are associated with the direct pathway (Menalled et al., 2002).

Although some studies report that neurodegeneration starts in the striatal matrix and continues to the striosomal neurons, others have reported the reverse progression (e.g., Hedreen \& Folstein, 1995). Tippett et al. (2007) observed that both directions of progression 
occur and that the heterogenic pattern is associated with the heterogeneity in clinical symptoms. Interestingly, the balance between striosomal and matrix loss was associated with the number of CAG repeats.

\subsubsection{The mutant huntingtin protein destabilizes cells}

Several studies have shown that the mutant huntingtin protein is neurotoxic. For example, phosphorylation of amino acids on the huntingtin protein reduces its toxicity $(\mathrm{Gu}$, et al., 2007). The precise pathway of toxicity is yet unknown, but evidence suggests that the mutant huntingtin protein interacts with DNA transcription factors, such as CREB (cAMP response element-binding protein) and dysregulates DNA transcription processes. In particular, there is evidence showing that the mutant huntingtin interferes selectively with transcription factors in the medium spiny neurons (Gomez, et al., 2006). In transgenic mice, Hebb et al. (2004) observed decreased levels of PDE10A mRNA before motor symptoms appear and Gomez et al. (2006) observed decreased levels of DARPP-32 mRNA in MSNs, but not in other DARPP-32 mRNA expressing tissue, such as the kidneys.

Several interacting intracellular pathways have been identified that are negatively affected by the mutant huntingtin protein (see for reviews, Cha, 2007; Luthi-Carter \& Cha, 2003). Apart from dysregulation of DNA transcription, mutant huntingtin has also been found to be associated with abnormal protein-protein interactions and with energy dysregulation in the mitochondria. The affected cells eventually enter a cascade resulting in apoptosis. It has been found that BDNF, which is needed for expression of DARPP32 (Ivkovic \& Ehrlich, 1999), and other neurotrophins are not only important in regulating the phenotype of striatal projection neurons (see for reviews, Pérez-Navarro, et al., 2000; Zuccato \& Cattaneo, 2007; Zuccato, et al., 2010), but also protected striatal neurons from the accelerated degeneration induced by huntingtin (Pérez-Navarro, et al., 2000).

\subsubsection{Compensatory mechanisms}

Substantial striatal atrophy precedes clinical motor symptoms (Aylward, 2006). In addition, cortical white matter decreases as the estimated years to disease onset decreases (Paulsen, et al., 2006; Stoffers, et al., 2010). However, cortical gray matter is above normal in pHD with large estimated years to onset and decreases to below normal the nearer the estimated onset time (Paulsen, et al., 2006; also observable in Stoffers et al., 2010). This increased gray matter volume is consistent with the increased functional activation in medial frontal areas (Beste, et al., 2007; Paulsen, et al., 2004), which is interpreted as reflecting a compensatory neurodevelopmental mechanism.

An important aspect to take into consideration with Huntington's Disease is that the individual has the CAG repeats from birth, but that the clinical symptoms are manifest at a much later stage in life. It is not inconceivable that during child development, the underresponsive indirect pathway is compensated for by the hyperdirect pathway. Using the hyperdirect pathway as a compensatory mechanism will affect cognitive tasks that depend on stopping an ongoing response or cognitive operation. This will be further addressed in sections 3.2.1 and 4.2. 


\subsubsection{Predicting disease onset}

Predicting the onset of HD is of great importance to the affected individuals and their families. As discussed by Aylward (2006), although detection of the mutated gene can be done at any time, treatment will not begin until the very earliest signs of change. The literature is somewhat biased with regard to what constitutes an early sign. Langbehn et al. (2004) obtained clinical data from 2913 individuals from 40 centers involved in the PREDICT-HD study. Using survival analyses which include the number of CAG repeats in symptomatic and presymptomatic individuals they derived a statistical model that predicts the disease onset given the number of CAG repeats and current age of the individual. Critical for conducting such an analysis is the need for an agreed upon endpoint. Langbehn et al. (2004) chose as the end point "the first-time neurological signs representing a permanent change from the normal state" (p. 268). This assumes that physician's accuracy in determining the clinical status of an individual is infallible. As Aylward (2006) discusses, this is seldom the case and much research remains needed to find objective biomarkers that help pinpoint the disease onset (Weir, et al., 2011). One such objective measure is striatal volume loss (Aylward, 2006). The loss of striatal neurons is a gradual process that starts around 10 years before the clinical onset and is associated with cognitive decline. In other words, one can discern a second earlier endpoint beyond which there is an accelerated loss of striatal volume and an increase in cognitive impairment. During the 10 year period, "presymptomatic" individuals are more prone to commit cognitive errors due to difficulty in concentration or inability to operate multiple complex tasks simultaneously. Although the acknowledgement of cognitive decline in presymptomatic individuals has attracted more cross-disciplinary research, it also comes with the fear that the presymptomatic individuals are at risk of committing preventable (and potentially fatal) accidents at the workplace. It is therefore critical to understand exactly what types of cognitive deficits coincide with striatal neuron loss and predict the onset of the classical symptoms by which HD is diagnosed.

There exists a strong regularity among trinucleotide disorders with the age of disease onset being an exponential function of number of trinucleotide repeats (Kaplan, et al., 2007; Walker, et al., 2007). Kaplan et al. (2007) proposed a model based on three assumptions. First, above a lower disease-related threshold, the trinucleotide repeats lead to damage that requires DNA repair processes. Second, these repair processes have a tendency to become error prone with more repeats, leaving the repaired DNA with even longer repeats. This repair-error-repair continues at an increasingly faster rate. Third, when the number of trinucleotide repeats reaches an upper critical threshold in a certain number of cells, the clinical symptoms of the disease become manifest. This simple model abstracts away from the various detailed molecular processes mentioned in the previous section. Nevertheless, it captures the exponential onset curve seen across trinucleotide repeat disorders and provides a statistical account of the correlations between disease progression and disease onset (the earlier the onset the faster the disease progression).

\subsection{Cognitive decline in Huntington's Disease}

The basal ganglia are involved in various cognitive processes (Aglioti, 1997; Grahn, et al., 2009; Heyder, et al., 2004) and therefore it is expected that neurodegeneration of striatal MSNs will have concomitant effects on cognitive processing, even in the absence of motor deficits. There is an abundance of studies investigating the cognitive decline in pHD and 
early stage HD employing various tasks and methods (e.g., Ho, et al., 2002, 2003; Lawrence, et al., 1996; Montoya, et al., 2008; Peretti, et al., 2008; Solomon, et al., 2007; Stout, et al., 2011).

Understanding the pattern of cognitive decline beyond the standard neuropsychological tests is vital. For example, many test measures do not differentiate between patient populations and most measures do not address compensatory mechanisms. A clinical solution is to use batteries of tests and combine the scores to improve overall diagnostic utility. A complementary solution, advocated here, is to combine tests that are sensitive to the specific cognitive mechanism(s) that is/are affected in the disorder. To do this, a very detailed understanding of the tests is required. For several tasks this is indeed the situation and much of the research in mathematical psychology and cognitive science is dedicated to understanding the cognitive operations that take place in a particular task. The measures that are accounted for are the usual primary measures, such as accuracy, response times, and higher moments of response time distributions, but also secondary measures, such as speed-accuracy tradeoff functions, conditional probabilities, and clustering. Some of those tasks have also been used extensively in conjunction with brain imaging and electrophysiological recordings, enriching our understanding of which brain structures process the information and when. Together with neuropsychological investigations of these tasks in $\mathrm{HD}$ and $\mathrm{pHD}$, a detailed picture emerges of the progression of cognitive decline that could be combined with objective biomarkers to not only answer the question "When will I get HD?", but also answer the question "Where am I in my neurocognitive journey?"

Space does not allow a thorough overview of the various tests used HD research and the reader is invited to consult the relevant articles for tests that are not discussed in the following sections and for further information about the tests that will be discussed.

\subsubsection{Flanker task: Evidence for compensatory mechanisms}

In this task, participants are required to respond to a target character that is presented on a computer screen. For example, if the target character is a right- or left-pointing arrow, the right of left response key needs to be depressed, respectively. The target character is flanked by distractor arrows on either side that are either pointing in the same (congruent condition) or opposite (incongruent condition) direction as the target arrow. The response time in the incongruent condition is slower than in the congruent condition, which is explained by the interference caused by the distractors. Huntington's patients are generally slower in this task, but do not show an abnormal interference effect (Beste, et al., 2008).

Apart from behavioral measures, Beste et al. $(2006,2007,2008)$ recorded electrophysiological responses and observed that compared to controls, HD showed reduced stimulus-related potentials (i.e., N1), slowed motor-related components (i.e., lateralized readiness potentials), and reduced error-related processing (i.e., Ne/ERN). Interestingly, the CAG-index of HD patients correlated with the size of the Ne/ERN response (Beste, et al., 2006). Compared to $\mathrm{HD}$, pHD showed stronger brain responses, but not more than controls, apart from errorrelated processing (Beste, et al., 2007, 2008). It has been suggested that pHD may exhibit compensatory activation in other brain regions (Feigin, et al., 2006; Paulsen, et al., 2004). The enhanced $\mathrm{Ne} / \mathrm{ERN}$ in $\mathrm{pHD}$ might indicate an upregulated hyperdirect pathway. 


\subsubsection{Memory: Seeing the trees through the forest}

There are many processes involved in memory and it is still uncertain which processes are differentially affected in HD. There is a myriad of theories about the role of the basal ganglia in learning and memory. In the interest of brevity, the reader is referred to the review articles that address research in animals and computational studies on long-term potentiation, depression and reinforcement learning (e.g., Da Cunha, et al., 2009; White, 1997). In a nutshell, the basal ganglia build stimulus-response mappings that provide the largest amount of reward. These types of learning can be considered procedural to the extent that no deliberate act of learning takes place other than valuating the stimulusresponse contingency. Here, the role of the basal ganglia in deliberate memory encoding and retrieval will be addressed.

With regard to memory encoding, the basal ganglia have been compared to a gatekeeper that allows entry of memoranda into working memory (McNab \& Klingberg, 2008). This gateway function is implemented by the disinhibition of the cortico-thalamic loop. Davelaar et al. (2005) showed how this gateway mechanism influences working memory updating and it is expected that this function is impaired in HD. At memory retrieval, Rohrer et al. (1999) showed that HD exhibit slow episodic retrieval, which in turn would lead to lowered total recall under speeded conditions. In a simulation model, Davelaar (2007) showed how degradation of the same gatekeeper pathway, the direct pathway, captures the slowed, but accurate memory performance.

\subsubsection{Random number generation}

For a task to be clinically useful, performance on the task should be stable and vary only in relation to the disease progression. That means that the ideal task does not show learning effects and is novel every time it is administered. Of course, from a clinician's point of view a task that requires electrophysiological recording or analyses of response time distributions is far from ideal. A task that requires two minutes and ticks the boxes is the random number generation task. In this task, the participant is required to produce one hundred digits from one to ten in a random fashion at a rate of one digit per second. This is relatively fast, but faster and slower rates have been used in the cognitive literature to understand the influence of production rate on executive functioning (Jahanshahi, et al., 2006; Towse, 1998). People are seldom truly random and the deviation from randomness can be captured with a variety of measures (Jahanshahi, et al., 2006; Towse \& Neil, 1998). People have a tendency to produce a sequence of digits that exhibits single-digit increments or decrements (e.g., "1, 2, $3 ", " 7,6,5 ")$. This tendency is captured quantitatively in the adjacency score and is calculated as the number of adjacent pairs divided by the total number of response pairs (see Towse \& Neil, 1998). In HD, counting in ones shows the greatest between group differences, with HD patients differing from $\mathrm{pHD}$ and controls, while there is no difference between the last two groups (Ho, et al., 2004).

\subsection{A classification scheme for tasks used in HD research}

Most of the cognitive deficits can be understood as a general inability to engage in or disengage from cognitive processing or where processing does occur it is slowed down. Based on considering a number of tasks and the results of simulations, the following 
classification scheme is proposed in which tasks are grouped in accordance with the required basal ganglia pathways.

Extra-basal ganglia (EBG) tasks - these are tasks that do not require the basal ganglia for much of the processing, other than as an output system. These are tasks such as simple and choice response time and are likely to be affected in later stages of HD. Despite the central role of working memory updating, it is still a form of output, albeit at the cognitive instead of motor level. Therefore, a task that only requires the gatekeeper pathway is an EBG task.

Intra-basal ganglia (IBG) tasks - these are tasks that are sensitive to the release of a recently chosen channel. Tasks that require a disengagement of selected information are random number generation and the use of retrieval cues in episodic memory tasks.

The direction of neuropathology, from striosomal to matrix or the reverse, was shown to be correlated with mood changes (Tippett, et al., 2007). It is assumed that the dysfunctional striosomal STR-SNc loop underlies this pattern and involves the reinforcement learning system. Thus depending on the precise pathology, category learning may be affected in $\mathrm{pHD}^{1}$. As responses in category learning end the respective trial, category learning tasks are also EBG tasks. This adds another dimension, namely the presence/absence of the necessity of reinforcement learning that can be crossed with the extra/intra-BG dimension, producing four types of tasks: $\mathrm{EBG}^{-}, \mathrm{EBG}^{+}, \mathrm{IBG}^{-}$, and $\mathrm{IBG}^{+}$. It should be stressed that classification of a task as an EBG- task, does not mean that no learning takes place. In fact, the flanker task would be classified as an EBG- task, but the observed sequential effects are signatures of associative learning (Davelaar \& Stevens, 2009). Therefore, great care should be taken to refer to the precise dependent measure that is being used. No example, of an $\mathrm{IBG}^{+}$task is given here, as no such task has been employed in HD. The simulations in section 4 address only EBG- and IBG- tasks (and task measures).

\section{Modeling Huntington's Disease}

In this section, results of simulation studies are presented that address cognitive decline before the clinical onset of HD. Details of most the models are available in the relevant publications, but critical features of existing models or novel details are presented where possible.

\subsection{Differential consequences of loss of D1- or D2-pathway}

Before presenting simulations of cognitive tasks, the influence of the direct and indirect pathways on the thalamic output needs to be clarified, as results are crucial in understanding the successes and failures of different tasks and task measures in detecting cognitive decline in pHD. Consider the simplified network in Fig. 2. When a single signal is presented to the network (see Fig. 3), the direct pathway releases the thalamic inhibition, whereas the indirect pathway stops the release. This results in a pulse as the thalamic output. When the integrity of the D2-pathway decreases, as in $\mathrm{pHD}$, the thalamus remains disinhibited for a longer time. When the D1-pathway is compromised, as in HD, the thalamic output is slowed.

1Stout et al., (2011) presented results showing no impairment on category learning in pHD. However, as task performance was not correlated with neuroanatomical data, the hypothesis of impaired reinforcement learning remains to be tested. 


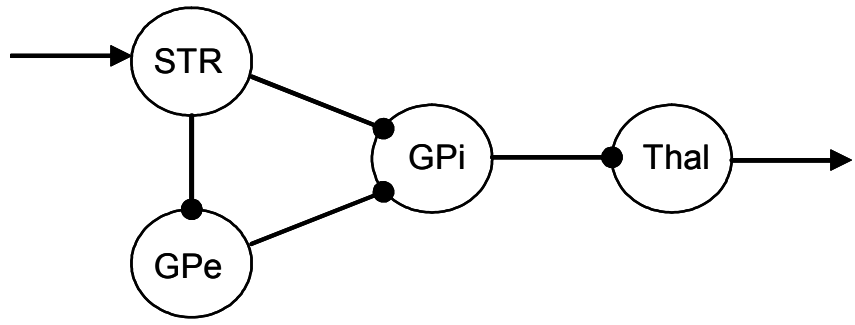

Fig. 2. Simplified basal ganglia network to address the consequences of loss of the D1- and D2-pathways.

When a sequence of signals is presented to the network, individual pulses can be discerned in the thalamic output. D2-pathway loss leads to interference in the ability to release the chosen channel, as seen in the middle column of Fig. 3. Thus, the continued disinhibition of the previous response interferes with the onset of the response to the next stimulus. This can be alleviated by compensation via the hyperdirect pathway. Loss of D1-receptor MSNs results in overall slower response onsets without interference.
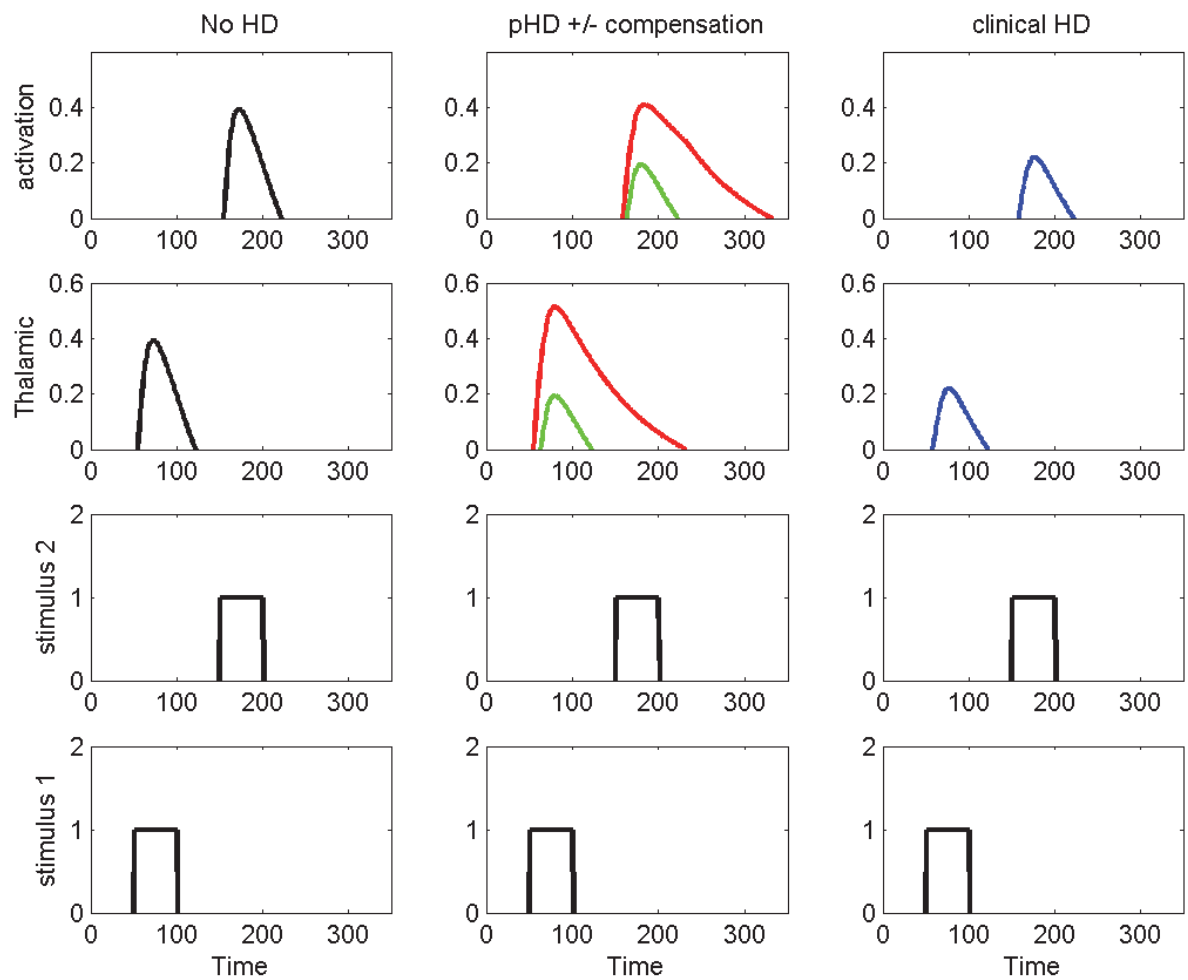

Fig. 3. The effect of loss of the D1- and D2-pathways in HD on thalamic output. First column: control situation. Middle column: pHD without (red lines) and with (green lines) compensation via the hyperdirect pathway. Last column: thalamic output in clinical HD. 


\subsection{Neural compensation for loss of D2-pathway: Evidence from flanker task}

As described in section 3.2.1, in the flanker task, stimuli made up of arrows (e.g., $<<<<<$, $<<><<)$ need to be categorized based on the central target character. In a series of papers, Beste et al. $(2006,2007,2008)$ presented various analyses of conflict processing in presymptomatic and symptomatic HD. Although the usual slow down in response times was observed for HD, this was absent in pHD. The critical data that will be focused on here are the $\mathrm{N} 2$ and the Ne/ERN components, both of which have been argued to reflect error-related controlled processing (Yeung \& Cohen, 2006). Both ERP components are calculated with respect to the response time, i.e., the individual trials are aligned to the time of response in each individual trial. The N2 is calculated as the difference between the ERP for correct incongruent trials and correct congruent trials ${ }^{2}$. The Ne/ERN is calculated as the difference between the erroneous incongruent trials and the correct incongruent trials.

\subsubsection{Method}

For this simulation, the model by Davelaar (2008) was used which implements the arrow flanker task. The model was developed to address the role of conflict monitoring in attentional control. In particular, the conflict was assumed to be monitored by medial frontal areas and this conflict influenced the amount of attention paid to filter out the distracting flanker characters. The model provided a good account of complete response time distributions, ERP latencies and fMRI BOLD responses. The details of the model are in Davelaar (2008). Here, one addition was made. The part of the model that represents the medial frontal areas which monitors the conflict signal was modulated by dopamine, while the input gradually decreases. Loss of D2-receptor MSNs is assumed to lead to increased sensitivity of cortical neurons. The resulting activation response functions with the nonmonotonic pattern of compensation are presented in Fig. 4 (left panel). This provides a dynamical control system by which the hyperdirect pathway takes over from the indirect pathway, which is one interpretation of the increased cingulate activation (Paulsen, et al., 2004).

\subsubsection{Results and discussion}

Table 1 shows the mean response times (with standard deviations in brackets) and accuracy for the two conditions for each simulated group. No effect of group is found on the behavioral measures. The ERP components, however, do differ among the groups. Compared to the no HD group the two pHD groups show larger N2 and Ne/ERN amplitudes, whereas the HD group shows a marked decreased amplitude (see Fig. 4). This pattern mirrors that of Beste et al. $(2006,2007,2008)$ for the Ne/ERN and is consistent with the putative compensatory mechanism of the cingulate cortex (Paulsen, et al. 2004).

\footnotetext{
${ }^{2}$ Beste et al. (2008) computed an N2 within incongruent trials. This is a different measure than that used in studies on cognitive control. Nevertheless, the simulation results with the standard N2 calculation is used and can be treated as a prediction by the model.
} 


\begin{tabular}{|l|l|l|l|l|}
\hline & No HD & "pHD far" & "pHD near" & "HD onset" \\
\hline RT - Congruent & $193(16)$ & $192(18)$ & $193(17)$ & $192(17)$ \\
\hline RT - Incongruent & $267(26)$ & $167(24)$ & $266(24)$ & $267(25)$ \\
\hline Accuracy - Congruent & $100 \%$ & $100 \%$ & $100 \%$ & $100 \%$ \\
\hline Accuracy - Incongruent & $89 \%$ & $87 \%$ & $88 \%$ & $88 \%$ \\
\hline N2-peak & 0.68 & 1.47 & 2.15 & 0.17 \\
\hline Ne/ERN-peak & 0.47 & 0.96 & 1.15 & 0.06 \\
\hline
\end{tabular}

Table 1. Results of flanker simulation with compensation via hyperdirect pathway. The stages of HD pathology are tentative, but the sequence follows the progressive loss of the D2-pathway.
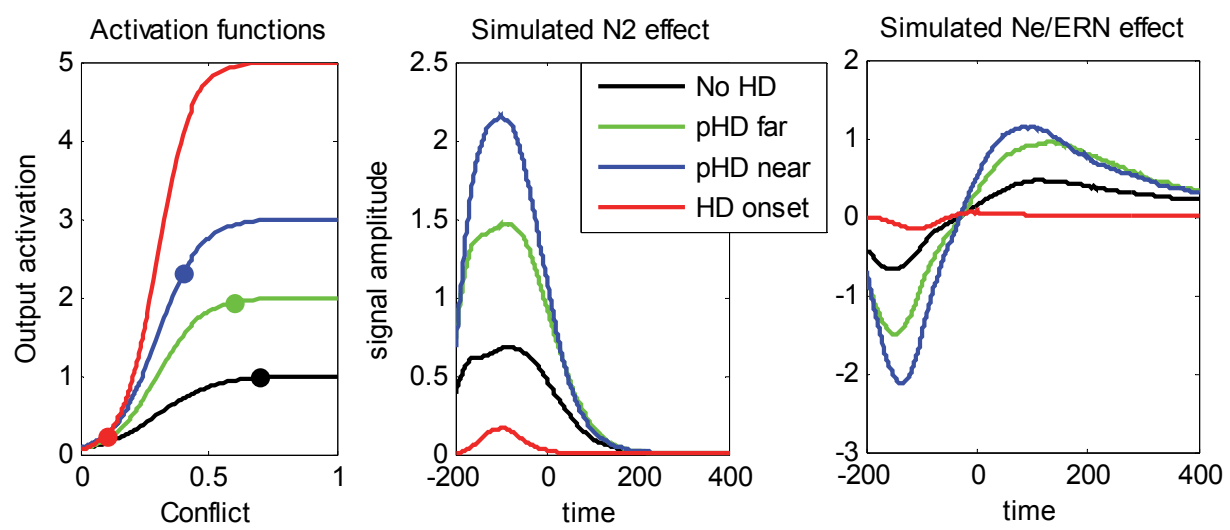

Fig. 4. Simulation results of the flanker task with compensation via the hyperdirect pathway. Left panel: illustration of how increased sensitivity of medial frontal areas together with decreased input leads to a nonmonotonic level of activation. Middle panel: simulation results for the N2-component. Right panel: simulation results for the Ne/ERN-component.

\subsection{Working memory capacity: The role of D1-pathway}

As mentioned in 3.2.2, the basal ganglia have been attributed a gatekeeper function (McNab \& Klingberg, 2008). Davelaar et al. (2005) showed how this gatekeeper function affects updating the contents of working memory. Yet, in neuropsychological studies, the memory span task is used to measure working memory capacity. In the following simulation, it is shown how capacity is affected by the D1-pathway. Note that the D2-pathway will not influence performance in this simulation as the cognitive process stops after an item is gated into working memory.

\subsubsection{Method}

The working memory model by Davelaar (Davelaar, 2007; Davelaar, et al., 2005) was used to simulate a memory span task. In this task, items are presented sequentially and need to be reported in the correct serial order. The capacity of the model is the maximum number of items that can be presented for which all items are reported in the correct serial order. To implement pHD, a $40 \%$ loss of the D2-pathway was chosen, while a further $50 \%$ loss of the D1-pathway was chosen to implement HD (cf. Glass, et al., 2000). 


\subsubsection{Results and discussion}

The results are as was expected with simulations of memory spans for controls, pHD, and $\mathrm{HD}$ of 3.88, 3.76, and 2.54, respectively. The gatekeeper pathway involves the D1-pathway and thus deficits in memory span are observable after onset of HD.

\subsection{Random number generation: Information sharing in D1-pathway}

The random number generation task has been shown to be sensitive to basal ganglia pathology and an ideal test for investigating executive functioning in neuropsychological patients. As mentioned in section 3.2.3, HD patients show more stereotyped behavior than pHD and controls, whereas pHD and controls do not differ. The following simulation provides an answer for this difference.

\subsubsection{Method}

The model is a working version of a network model proposed by R. G. Brown et al. (2000, see also Jahanshahi, et al., 1998) and is based on the memory model of sequential retrieval by Davelaar (2007) that is augmented with a semantic memory system containing the digits in an associative fashion. That is, the digit 3 was associated strongly with the digits 2 and 4 and less strongly with digits 1 and 5. This gradient was used for all digits. The actual simulation uses an abstracted version to speed up the computer time, but the model makes use of two cortico-basal loops (see Fig. 5). The first is the loop that selects an individual digit, while the second loop selects the motor program associated with this digit. From Fig. 5 it can be seen that the associative structure corresponds to each digit being associated with multiple motor programs. Neuropsychological investigations on frontal patients using a speeded naming task are suggestive of such architecture (Thompson-Schill \& Botvinick, 2006).

Initially, all digits are activated and when the digit is selected it receives inhibition, leading to a lower likelihood of selecting the same digit in succession. This implements the ubiquitous repetition avoidance (Towse, 1998). The remaining activations may be selected when they have not yet decayed to baseline. The reason for separating the selection of the digit and the selection of the motor program, is that a single loop has been shown to (incorrectly) predict a decrease in stereotyped behavior with increasing production rate (Davelaar, 2004) instead of an increase (Towse, 1998). This process continues until a hundred digits have been reported.

Loss of the indirect pathway was modeled by decreasing the rate at which selection suppression and recovery takes place. Loss of the direct pathway was modeled by increasing the threshold for entry in the set of selection candidates. This loss was implemented in both loops simultaneously.

\subsubsection{Results and discussion}

Fig. 6 (left panel) shows the distribution of first-order differences at three levels of the HD progression. The distributions show clear repetition avoidance and a tendency to report adjacent digits. Importantly, this counting behavior is larger for the HD simulation than for the pHD and control simulations. The right panel in Fig. 6 shows the adjacency score as 
standardized score in which a zero score means perfect random generation (see Towse, 1998). In the model, the adjacency score is not influenced by loss of the indirect pathway, but is sensitive to loss of neurons in the direct pathway.

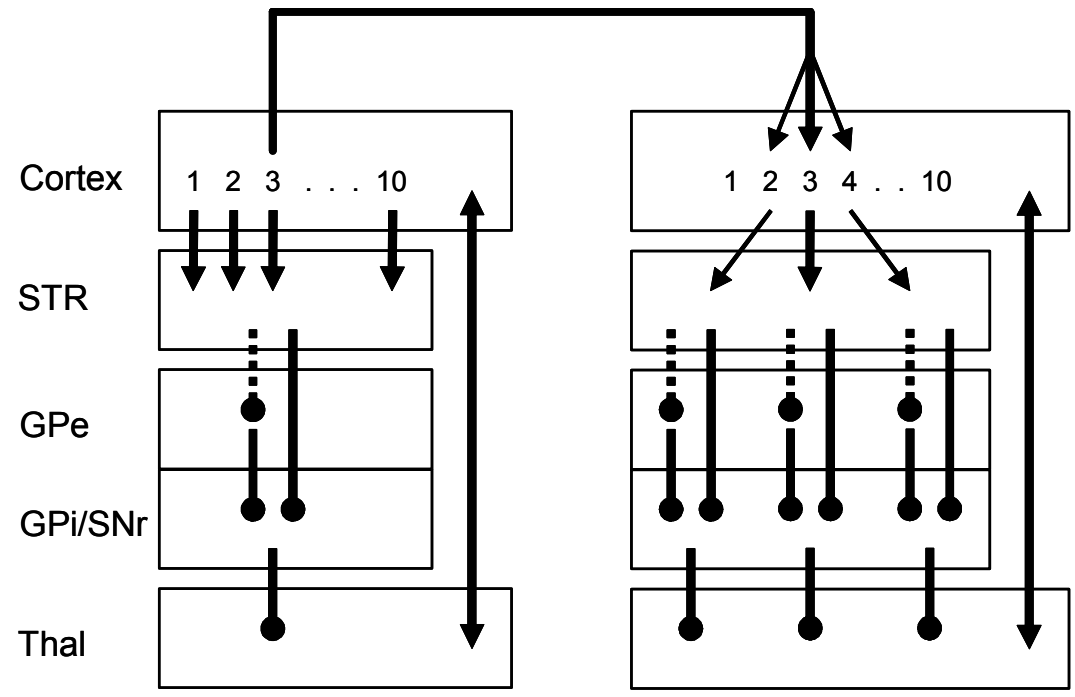

First selection loop

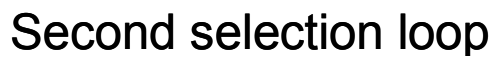

Fig. 5. Basal ganglia model of the random number generation task involving two selection loops. The first loop selects the digits from memory and the second loop selects the associated motor response. The associative network resides in the cortico-cortico connections. For simplicity the STN and other within basal ganglia connections have been omitted.
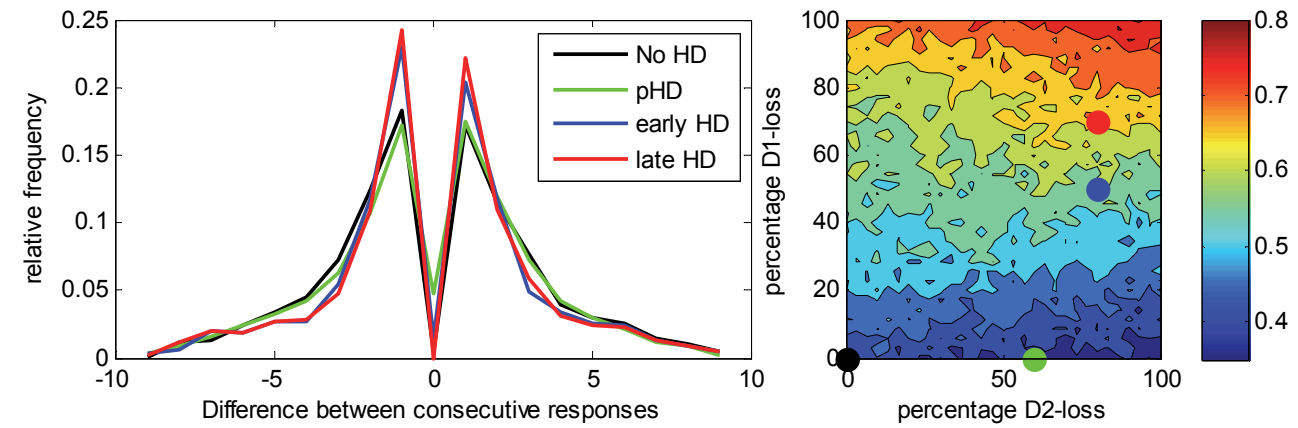

Fig. 6. Results of the simulation on random number generation. Left: distribution of firstorder differences. Distributions of the highlighted combinations of D1/D2-loss in the right panel are shown. Right: standardized adjacency scores at various levels of loss in D1- and D2-pathways. Percentage loss is approximately corresponding to reports by Glass et al. (2000). 


\subsection{Memory strategies: A window into D2-pathway loss}

The simulations showed that HD pathology influence memory only via the direct pathway which would mean that $\mathrm{pHD}$ would not involve memory deficits. Yet, several studies have shown various memory deficits in pHD (Solomon, et al., 2007; Stout, et al., 2011). Critically, Stout et al. (2011) showed that performance on the Hopkins Verbal Learning Test (HVLT) still predicted the probability of disease onset in 5 years after controlling for the standard Unified Huntington's Disease Rating Scale (UHDRS) Motor Score. How to reconcile these opposing views?

The model of random number generation is an example of a system that first selects a cue and then uses this cue to select candidates for output. This is closely related to the use of cues in memory tasks and the HVLT in particular. In this task, participants are presented with items for immediate or delayed free recall. The sequence of items consists of four words from three categories. The strategic use of cues, such as category labels improves overall recall performance. Therefore it is better to first select a cue and then use the cue to select items from memory than to directly try to retrieve the items from memory. This extra step would involve the first selection loop in the random generation model. With the HVLT, the selection is not random, but involves choosing which of the three category labels to use. Once the category label is chosen, words that belong to that category and have episodic contextual list information will be selected as candidates for retrieval.

This two-stage retrieval process requires the indirect pathway to let go of the chosen cue when it is not needed anymore. It is expected that $\mathrm{pHD}$ would have difficulty in performing this cognitive operation. Even though loss of the indirect pathway did not influence the adjacency score in random number generation, none of the measures used in the literature on random number generation is specifically designed to capture the operation of the first selection loop. In the verbal learning test, not being able to disengage from a cue will limit the total recall performance and thus pHD should show selective memory impairment.

Duff et al. (2010) discuss the possibility of dividing pHD into an amnestic and a nonamnestic group. The measure used to distinguish this is the total recall on the HVLT together with scores from other tasks, with subnormal performance on any non-memory resulting in a nonamnestic categorization. Assuming that an amnestic and nonamnestic variant of pHD exist, how does this fit with the modeling framework? To answer this question, it needs to be realized that none of the verbal learning tests control for strategy use. That is, some individuals may use memory strategies, such as semantic retrieval cues, whereas others do not. Only when cues are being utilized will the inability to disengage from cues be observed. Thus, individuals that are classified as amnestic pHD might be those who tend to use optimal memory strategies. In longitudinal studies, this will be observed as a high performing affected individual declining faster than low performing individuals. The final simulation is designed to demonstrate this pattern.

\subsubsection{Method}

A computational model of chunking is used that accounts for idiosyncratic chunking behavior and is a combination of the random generation model and the search of associative memory (SAM; Raaijmakers \& Shiffrin, 1981). To model the longitudinal trajectory of pHD the probability of disengaging a cue was decreased over the course of $\mathrm{pHD}$. To increase the 
performance for the simulation of nonamnestic $\mathrm{pHD}$, the short-term capacity was increased. This is justified under the assumption that people choose to either focus more during encoding or work harder during retrieval. Note that in this simulation the selection threshold stays fixed. In the model for the random number generation and in a simulation study on recall latencies in HD (Davelaar, 2007), the threshold increase relates to the stage after clinical onset. In simulation studies that focus on the longitudinal trajectory of memory impairment after disease onset this parameter would need to be increased as the disease progresses.

\subsubsection{Results and discussion}

The results are shown in Fig. 7. As discussed above, total recall is better when memory cues are being used. When no strategy is used involving sequential cue retrieval, the memory performance stays relatively constant and fluctuations are within normal ranges. When the retrieval strategy is used, there is an advantage at the beginning of the pHD, which turns into a disadvantage the nearer the disease onset time. These results partly explain why memory tests are good short-term predictors of disease onset (Stout, et al., 2011).

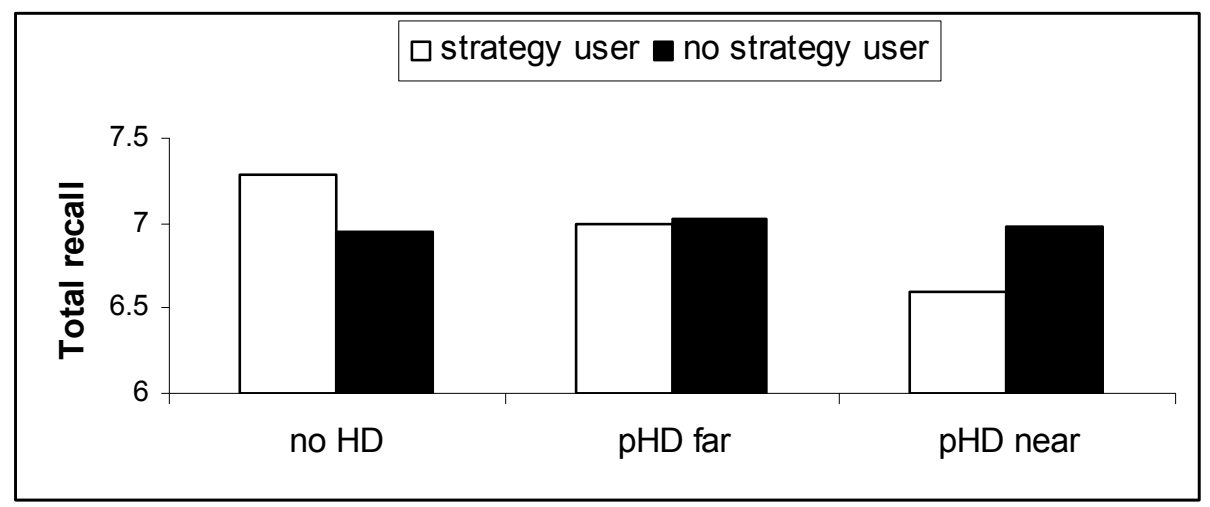

Fig. 7. Simulation results of a verbal learning experiment.

At each stage, until just prior to disease onset, the total recall performance leads to misclassification of those individuals who use memory cues to aid their retrieval. The choice of using memory cues is voluntary and thus an individual (e.g., no strategy user) may be classified as amnestic at an early stage and nonamnestic at a later time. This is possible explanation for the erratic pattern presented in Duff, et al. (2010).

\section{General discussion}

This chapter has presented several simulation results regarding the cognitive decline in symptomatic and presymptomatic HD. The architectures of the computational models respect the known functional neuroanatomy and neuropathology in HD. The results showed how compensation via the hyperdirect pathway influences performance in the flanker task (section 4.2) and why memory span (section 4.3) and random number generation (section 4.4) only show a decline when the D1-pathway is affected. Finally, with 
regard to mild cognitive impairment in pHD with amnestic and nonamnestic variants, simulations verify the MCI variants and provide an intuition on how to maximize the differential classification (section 4.5).

As can be seen in Fig. 3, loss of D2-receptor MSNs result in increased times to disengage the chosen response. Only when D1-receptor MSNs are lost, does the response time increase. This has important implications for longitudinal studies that aim to assess the cognitive decline in pHD. With the assumption that pHD is mainly associated with loss of the indirect pathway (cf. Glass, et al., 2000), it is expected that tasks that depend on disengagement after a choice are ideal candidates for screening. However, compensatory mechanisms may mask the degeneration of the indirect pathway, making it difficult to observe deficits in cognitive and motor stopping behavior until near the onset of HD.

Yet, failure to disengage after a choice can be observed in situations where normally a maintained focus would be beneficial. In section 4.5, memory retrieval was addressed, which involves a two-stage process, with selection of a cue followed by selection of an item. Failure to disengage from the cue impacted on total recall performance. The strategy of using retrieval cues is under voluntary control, leading to variability among individuals in the likelihood of using memory strategies. It is likely that some pHD individuals will attempt using retrieval cues and some do not. For pHD individuals who do attempt cued retrieval, a memory enhancement is expected when they are far removed from the disease onset, but a memory deficit is expected when they are closer to the disease onset.

The division of mild cognitive impairment in pHD into an amnestic and a nonamnestic variant Duff et al. (2010) would map onto those who do and those who do not attempt memory strategies. In addition, the choice of using a memory strategy is unlikely to involve any components that are measured using the UHDRS Motor Score. This would explain why the HVLT has predictive power after controlling for UHDRS Motor Score (Stout, et al., 2011). Currently, these are still preliminary predictions that need to be confirmed in empirical studies.

Apart from the choice of tasks and task measures used in studies and the classification of presymptomatic $\mathrm{HD}$, there is a rich understanding of the neuropathology and its consequences for motor behavior. At this level, detailed computational models of the basal ganglia can be employed to extrapolate back in time to see how the deficit in motor behavior unfolds from early pHD to HD. With the added assumption that the cognitive loops undergo the same changes, albeit at a different sensitivity, the lifetime approach can be used to identify tasks that are sensitive to pHD status. These types of models therefore bridge the detailed findings about the progression of the neuropathology with the overall cognitive outcome.

Computational models are in essence complex statistical models. Conventional statistical tests may take simplifying assumptions, such as simplified functional forms, normally-distributed variance, equality of variance, and many more. Computational models are not developed within the framework of statistical theory, but can be developed to correspond to functional neuroanatomical and neuropathological reality. In this way, neurally plausible computational models can be used to extrapolate back in time and are arguably the best reality-grounded statistical models we have. Verification of the model predictions is still needed. Studies such as PREDICT-HD (e.g., Stout, et al., 2011) employed tasks for which computational models exist and therefore the required database is present to provide the final impetus to include computational modeling as an additional methodology in HD research. 
Throughout this chapter, the critical data that grounded the models was the observation that disease onset coincides with loss of D1-receptor MSNs. The proposed classification of tasks is based on the tasks' reliance of the D1- and D2-pathways and associative learning (section 3.3). The power of the tasks to predict disease onset depends on the precise profile of D1MSN loss. The computational approach requires reliable and objective biomarkers. Once these have been validated, new HD-specific cognitive batteries can be developed that predict the probability of disease onset even after controlling for UHDRS Motor Score. This will further improve the clinician's ability to grade a person's cognitive decline and support the affected individual and families.

\section{Conclusion}

This chapter presented computer simulations of tasks that have been used in the cognitive research on presymptomatic and symptomatic HD. The models were developed to be close to the underlying neurobiological factors that drive the disease. The models are still premature, but have the potential to contribute to intervention strategies. To bring forth tangible results, interdisciplinary research is vital and will benefit those with HD, those who are aware they will get HD, and the society who needs to prepare and provide care for those affected. Such a research program remains for the future. This chapter presented a first stage, demonstrating the ability of neuroanatomically grounded computational models to capture cognitive performance and decline in presymptomatic Huntington's Disease.

\section{References}

Aglioti, S. (1997). The role of the thalamus and basal ganglia in human cognition. Journal of Neurolinguistics, 10, 255-265, ISSN 0911-6044

Albin, R. L., Young, A. B., \& Penney, J. B. (1989). The functional anatomy of basal ganglia disorders. Trends in Neurosciences, 12, 366-375, ISSN 0166-2236

Alexander, G. E., \& Crutcher, M. D. (1990). Functional architecture of basal ganglia circuits: neural substrates of parallel processing. Trends in Neurosciences, 13, 266-271, ISSN 0166-2236

Aylward, E. H. (2006). Change in MRI striatal volumes as a biomarker in preclinical Huntington's disease. Brain Research Bulletin, 72, 152-158, ISSN 0361-9230

Bar-Gad, I., Morris, G., \& Bergman, H. (2003). Information processing, dimensionality reduction and reinforcement learning in the basal ganglia. Progress in Neurobiology, 71, 439-473, ISSN 0301-0082

Bergman, H., Feingold, A., Nini, A., Raz, A., Slovin, H., Abeles, M., \& Vaadia, W. (1998). Physiological aspects of information processing in the basal ganglia of normal and parkinsonian primates. Trends in Neurosciences, 21, 32-38, ISSN 0166-2236

Berns, G. S., \& Sejnowski, T. J. (1998). A computational model of how the basal ganglia produce sequences. Journal of Cognitive Neuroscience, 10, 108-121, ISSN 0898-929X

Beste, C., Saft, C., Andrich, J., Gold, R., \& Falkenstein, M. (2006). Error processing in Huntington's disease. PLoS ONE, 1, e86. doi: 10.1371/journal.pone.0000086, ISSN 1932-6203

Beste, C., Saft, C., Andrich, J., Gold, R., \& Falkenstein, M. (2008). Stimulus-response compatibility in Huntington's disease: a cognitive-neurophysiological analysis. Journal of Neurophysiology, 99, 1213-1223, ISSN 0022-3077 
Beste, C., Saft, C., Yordanova, J., Andrich, J., Gold, R., Falkenstein, M., \& Kolev, V. (2007). Functional compensation or pathology in cortico-subcortical interactions in preclinical Huntington's disease? Neuropsychologia, 45, 2922-2930, ISSN 0028-3932

Brown, J., Bullock, D., \& Grossberg, S. (1999). How the basal ganglia use parallel excitatory and inhibitory learning pathways to selectively respond to unexpected rewarded cues. Journal of Neuroscience, 19, 10502-10511, ISSN 0270-6474

Brown, R. G., Soliveri, P., \& Jahanshahi, M. (1998). Random response generation in Parkinson's disease and normal controls. Executive processes and the role of the prefrontal cortex. Neuropsychologia, 36, 1355-1362, ISSN 0028-3932

Bullock, D. (2004). Adaptive neural models of queuing and timing in fluent action. Trends in Cognitive Sciences, 8, 426-433, ISSN 1364-6613

Bullock, D., Tan, C. O., \& John, Y. J. (2009). Computational perspectives on forebrain microcircuits implicated in reinforcement learning, action selection, and cognitive control. Neural Networks, 22, 757-765, ISSN 0893-6080

Cha, J. J. (2007). Transcriptional signatures in Huntington's disease. Progress in Neurobiology, 83, 228-248, ISSN 0301-0082

Chevalier, G., \& Deniau, J. M. (1990). Disinhibition as a basic process in the expression of striatal functions. Trends in Neurosciences, 13, 277-280, ISSN 0166-2236

Cicchetti, F., Prensa, L., Wu, Y., \& Parent, A. (2000). Chemical anatomy of striatal interneurons in normal individuals and in patients with Huntington's disease. Brain Research Reviews, 34, 80-101, ISSN 0165-0173

Cohen, M. X., \& Frank, M. J. (2009). Neurocomputational models of basal ganglia function in learning, memory and choice. Behavioural Brain Research, 199, 141-156, ISSN 0166-4328

Da Cunha, C., Wietzikoski, E. C., Dombrowski, P., Bortolanza, M., Santos, L. M., Boschen, S. L., \& Miyoshi, E. (2009). Learning processing in the basal ganglia: a mosaic of broken mirrors. Behavioural Brain Research, 199, 157-170, ISSN 0166-4328

Davelaar, E. J., Goshen-Gottstein, Y., Ashkenazi, A., Haarmann, H. J., \& Usher, M. (2005). The demise of short-term memory revisited: empirical and computational investigations of recency effects. Psychological Review, 112, 3-42, ISSN 0033-295X

Davelaar, E. J. (2004). Random generation of items from memory: empirical and computational explorations. Poster presented at the 2nd International Conference on Working Memory, Kyoto, Japan.

Davelaar, E. J. (2007). Sequential retrieval and inhibition of parallel (re)activated representations: a neurocomputational comparison of competitive queuing and resampling models. Adaptive Behavior, 15, 51-71, ISSN 1059-7123

Davelaar, E. J. (2008). A computational study of conflict-monitoring at two levels of processing: reaction time distributional analyses and hemodynamic responses. Brain Research, 1202, 109-119, ISSN 0006-8993

Davelaar, E. J., \& Stevens, J. (2009). Sequential dependencies in the Eriksen flanker task: a direct comparison of two competing accounts. Psychonomic Bulletin E Review, 16, 121-126, ISSN 1069-9384

DeLong, M., \& Wichmann, T. (2009). Update on models of basal ganglia function and dysfunction. Parkinsonism and Related Disorders, 1553, S237-S240, ISSN 1353-8020

Doya, K. (2007). Reinforcement learning: Computational theory and biological mechanisms. HFSP Journal, 1, 30-40.

Duff, K., Paulsen, J., Mills, J., Beglinger, L. J., Moser, D. J., Smith, M. M., Langbehn, D., Stout, J., Queller, S., \& Harrington, D. L. (2010). Mild cognitive impairment in prediagnosed Huntington disease. Neurology, 75, 500-507, ISSN 0028-3878 
Eblen, F., \& Graybiel, A. M. (1995). Highly restricted origin of prefrontal cortical inputs to striosomes in the macaque monkey. Journal of Neuroscience, 15, 5999-6013, ISSN 0270-6474

Feigin, A., Tang, C., Ma, Y., Mattis, P., Zgaljardic, D., Guttman, M., Paulsen, J. S., Dhawan, V., Eidelberg, D. (2007). Thalamic metabolism and symptom onset in preclinical Huntington's disease. Brain, 130, 2858-2867, ISSN 0006-8950

Frank, M. J. (2006). Hold your horses: a dynamic computational role for the subthalamic nucleus in decision making. Neural Networks, 19, 1120-1136, ISSN 0893-6080

Frank, M. J., Loughry, B., \& O'Reilly, R. C. (2001). Interactions between the frontal cortex and basal ganglia in working memory: a computational model. Cognitive, Affective, and Behavioral Neuroscience, 1, 137-160, ISSN 1530-7026

Frank, M. J., \& Claus, E. D. (2006). Anatomy of a decision: striato-orbitofrontal interactions in reinforcement learning, decision making, and reversal. Psychological Review, 113, 300-326, ISSN 0033-295X

Glass, M., Dragunow, M., \& Faull, R. L. M. (2000). The pattern of neurodegeneration in Huntington's disease: a comparative study of cannabinoid, dopamine, adenosine and GABAA receptor alterations in the human basal ganglia in Huntington's disease. Neuroscience, 97, 505-519, ISSN 0306-4522

Gomez, G. T., Hu, H., McCaw, E. A., \& Denovan-Wright, E. M. (2006). Brain-specific factors in combination with mutant huntingtin induce gene-specific transcriptional dysregulation. Molecular and Cellular Neuroscience, 31, 661-675, ISSN 1044-7431

Grahn, J. A., Parkinson, J. A., \& Owen, A. M. (2009). The role of the basal ganglia in learning and memory: neuropsychological studies. Behavioural Brain Research, 199, 53-60, ISSN 0166-4328

Graybiel, A. M. (1991). Basal ganglia - input, neural activity, and relation to the cortex. Current Opinion in Neurobiology, 1, 644-651, ISSN 0959-4388

Graybiel, A. M. (1995). Building action repertoires: memory and learning functions of the basal ganglia. Current Opinion in Neurobiology, 5, 733-741, ISSN 0959-4388

Graybiel, A. M. (2005). The basal ganglia: learning new tricks and loving it. Current Opinion in Neurobiology, 15, 638-644, ISSN 0959-4388

Gu, X., Greiner, E. R., Mishra, R., Kodali, R., Osmand, A., Finkbeiner, S., Steffan, J. S., Thompson, L. M., Wetzel, R., \& Yang, X. W. (2009). Serines 13 and 16 are critical determinants of full-length human mutant huntingtin induced disease pathogenesis in HD mice. Neuron, 64, 828-840, ISSN 0896-6273

Gurney, K., Prescott, T. J., \& Redgrave, P. (2001). A computational model of action selection in the basal ganglia II: analysis and simulation of behaviour. Biological Cybernetics, 84, 411-423, ISSN 0340-1200

Guthrie, M., Myers, C. E., \& Gluck, M. A. (2009). A neurocomputational model of tonic and phasic dopamine in action selection: a comparison with cognitive deficits in Parkinson's disease. Behavioural Brain Research, 200, 48-59, ISSN 0166-4328

Hebb, A. L. O., Robertson, H. A., \& Denovan-Wright, E. M. (2004). Striatal phosphadiesterase mRNA and protein levels are reduced in Huntington's disease transgenic mice prior to the onset of motor symptoms. Neuroscience, 123, 967-981, ISSN 0306-4522

Hedreen, J. C., \& Folstein, S. E. (1995). Early loss of neostriatal striosome neurons in Huntington's disease. Journal of Neuropathology and Experimental Neurology, 54,105120, ISSN 0022-3069 
Heyder, K., Suchan, B., \& Daum, I. (2004). Cortico-subcortical contributions to executive control. Acta Psychologica, 115, 271-289, ISSN 0001-6918

Ho, A. K., Sahakian, B. J., Brown, R. G., Barker, R. A., Hodges, J. R., Ané, M., Snowden, J., Thompson, J., Esmonde, T., Gentry, R., Moore, J. W., \& Bodner, T. (2003). Profile of cognitive progression in early Huntington's disease. Neurology, 61, 1702-1706, 00283878

Ho, A. K., Sahakian, B. J., Robbins, T. W., \& Barker, R. A. (2004). Random number generation in patients with symptomatic and presymptomatic Huntington's disease. Cognitive and Behavioral Neurology, 17, 208-212, ISSN 1543-3633

Ho, A. K., Sahakian, B. J., Robbins, T. W., Barker, R. A., Rosser, A. E., \& Hodges, J. R. (2002). Verbal fluency in Huntington's disease: a longitudinal analysis of phonemic and semantic clustering and switching. Neuropsychologia, 40, 1277-1284, ISSN 0028-3932

Houk, J.C., \& Wise, S. P. (1995). Distributed modular architectures linking basal ganglia, cerebellum, and cerebral cortex: their role in planning and controlling action. Cerebral Cortex, 5, 95-110, ISSN 1047-3211

Humphries, M. D., Stewart, R. D., \& Gurney, K. N. (2006). A physiologically plausible model of action selection and oscillatory activity in the basal ganglia. Journal of Neuroscience, 26, 12921-12942, ISSN 0270-6474

The Huntington's Disease Collaborative Research Group (1993). A novel gene containing a trinucleotide repeat that is expanded and unstable on Huntington's disease chromosomes. Cell, 72, 971-983, ISSN 0092-8674

Ivkovic, S., \& Ehrlich, M. E. (1999). Expression of the striatal DARPP-32/ARPP-21 phenotype in GABAergic neurons requires neurotrophins in vivo and in vitro. Journal of Neuroscience, 19, 5409-5419, ISSN 0270-6474

Jahanshahi, M., Saleem, T., Jo, A. K., Dirnberger, G., \& Fuller, R. (2006). Random number generation as an index of controlled processing. Neuropsychology, 20, 391-399, ISSN 0894-4105

Jahanshahi, M., Profice, P., Brown, R. G., Ridding, M. C., Dirnberger, G., \& Rothwell, J. C. (1998). The effects of transcranial magnetic stimulation over the dorsolateral prefrontal cortex on suppression of habitual counting during random number generation. Brain, 121, 1533-1544, ISSN 0006-8950

Joel, D., \& Weiner, I. (1994). The organization of the basal ganglia-thalamocortical circuits: open interconnected rather than closed segregated. Neuroscience, 63, 363-379, ISSN 0306-4522

Joyce, J. N., Sapp, D. W., Marshall, J. F. (1986). Human striatal dopamine receptors are organized in compartments. Proceedings of the National Academy of Sciences, 83, 80028006, ISSN 1091-6490

Kaplan, S., Itzkovitz, S., \& Shapiro, E. (2007). A universal mechanism ties genotype to phenotype in trinucleotide diseases. PLoS Computational Biology, 3, e235. doi: 10.1371/journal.pcbi.0030235, ISSN 1553-734X

Langbehn, D. R., Brinkman, R. R., Gaulsh, D., Paulsen, J. S., \& Hayden, M. R. (2004). A new model for prediction of the age of onset and penetrance for Huntington's disease based on CAG length. Clinical Genetics, 65, 267-277, ISSN 0009-9163

Lawrence, A. D., Sahakian, B. J., Hodges, J. R., Rosser, A. E., Lange, K. W., \& Robbins, T. W. (1996). Executive and mnemonic functions in early Huntington's disease. Brain, 119, 1633-1645, ISSN 0006-8950 
Lévesque, M., \& Parent, A. (2005). The striatofugal fiber system in primates: a reevaluation of its organization based on single-axon tracing studies. Proceedings of the National Academy of Sciences, 102, 11888-11893, ISSN 1091-6490

Luthi-Carter, R., \& Cha, J. J. (2003). Mechanisms of transcriptional dysregulation in Huntington 's disease. Clinical Neuroscience Research, 3, 165-177, ISSN 1566-2772

Manninen, T., Hituri, K., Kotaleski, J. H., Blackwell, K. T., \& Linne, M. (2010). Postsynaptic signal transduction models of long-term potentiation and depression. Frontiers in Computational Neuroscience, 4, 152. doi: 10.3389/fncom.2010.00152, ISSN 16625188

McNab, F., \& Klingberg, T. (2008). Prefrontal cortex and basal ganglia control access to working memory. Nature Neuroscience, 11, 103-107, ISSN 1097-6256

Menalled, L. B., Sison, J. D., Wu, Y., Olivieri, M., Li, X., Li, H., Zeitlin, S., \& Chesselet, M. (2002). Early motor dysfunction and striosomal distribution of huntingtin microaggregates in Huntington's disease knock-in mice. Journal of Neuroscience, 22, 8266-8276, ISSN 0270-6474

Monchi, O., Taylor, J. G., \& Dagher, A. (2000). A neural model of working memory processes in normal subjects, Parkinson's disease and schizophrenia for fMRI design and predictions. Neural Networks, 13, 953-973, ISSN 0893-6080

Montoya, A., Price, B. H., Menear, M., \& Lepage, M. (2006). Brain imaging and cognitive dysfunctions in Huntington's disease. Journal of Psychiatry and Neuroscience, 31, 2129, ISSN 1180-4882

Nambu, A. (2011). Somatotopic organization of the primate basal ganglia. Frontiers in Neuroanatomy 5, 26. doi: 10.3389/fnana.2011.00026, ISSN 1662-5129

Nambu, A., Tokuno, H., \& Takada, M. (2002). Functional significance of the corticosubthalamo-pallidal 'hyperdirect' pathway. Neuroscience Research, 43, 111-117, ISSN 0168-0102

Nambu, A., Tokuno, H., Hamada, I., Kita, H., Imanishi, M., Akazawa, T., Ikeuchi, Y., \& Hasegawa, N. (2000). Excitatory cortical inputs to pallidal neurons via the subthalamic nucleus in the monkey. Journal of Neurophysiology, 84, 289-300, ISSN 0022-3077

Paulsen, J. S., Magnotta, V. A., Mikos, A. E., Paulson, H. L., Penziner, E., Andreasen, N. C., \& Nopoulos, P. C. (2006). Brain structure in preclinical Huntington's disease. Biological Psychiatry, 59, 57-63, ISSN 0006-3223

Paulsen, J. S., Zimbelman, J. L., Hinton, S. C., Langbehn, D. R., Leveroni, C. L., Benjamin, M. L., Reynolds, N. C., Rao, S. M. (2004). fMRI biomarker of early neuronal dysfunction in presymptomatic Huntington's disease. American Journal of Neuroradiology, 25, 1715-1721, ISSN 0195-6108

Peretti, C., Ferreri, F., Blanchard, F., Bakchine, S., Peretti, C. R., Dobrescu, A., Chouinard, V., \& Chouinard, G. (2008). Normal and pathological aging of attention in presymptomatic Huntington's, Huntington's and Alzheimer's disease, and nondemented elderly subjects. Psychotherapy and Psychosomatics, 77, 139-146, ISSN 0033-3190

Pérez-Navarro, E., Canudas, A. M., Åkerud, P., Alberech, J., \& Arenas, E. (2000). Brainderived neurotrophic factor, neurotrophin-3, and neurotrophin-4/5 prevent the death of striatal projection neurons in a rodent model of Huntington's disease. Journal of Neurochemistry, 75, 2190-2199, ISSN 1471-4159 
Perreault, M. L., Hasbi, A., O’Dowd, B. F., \& George, S. R. (2011). The dopamine D1-D2 receptor heteromer in striatal medium spiny neurons: evidence for a third distinct neuronal pathway in basal ganglia. Frontiers in Neuroanatomy, 5, 31 doi:10.3389/fnana.2011.00031, ISSN 1662-5129

Rohrer, D., Salmon, D. P., Wixted, J. T., \& Paulsen, J. S. (1999). The disparate effects of Alzheimer's disease and Huntington's disease on semantic memory. Neuropsychology, 13, 381-388, ISSN 0894-4105

Raaijmakers, J. G. W., \& Shiffrin, R. M. (1981). Search of associative memory. Psychological Review, 88, 93-134, ISSN 0033-295X

Solomon, A. C., Stout, J. C., Johnson, S. A., Langbehn, D. R., Aylward, E. H., Brandt, J., Ross, C. A., Beglinger, L., Hayden, M. R., Kieburtz, K., Kayson, E., Julian-Baros, E., Duff, K., Guttman, M., Nance, M., Oakes, D., Shoulson, I., Penziner, E., \& Paulsen, J. S. (2007). Verbal episodic memory declines prior to diagnosis in Huntington's disease. Neuropsychologia, 45, 1767-1776, ISSN 0028-3932

Stoffers, D., Sheldon, S., Kuperman, J. M., Goldstein, J., Corey-Bloom, J., Aron, A. R. (2010). Contrasting gray and white matter changes in preclinical Huntington's disease. Neurology, 74, 1208-1216, ISSN 0028-3878

Stout, J. C., Paulsen, J. S., Queller, S., Solomon, A. C., Whitlock, K. B., Campbell, J. C., Carlozzi, N., Duff, K., Beglinger, Langbehn, D. R., Johnson, S. A., Biglan, K. M., Aylward, E. H. (2011). Neurocognitive signs in prodromal Huntington disease. Neuropsychology, 25, 1-14, ISSN 0894-4105

Thompson-Schill, S. L., \& Botvinick, M. M. (2006). Resolving conflict: A response to Martin and Cheng (2006). Psychonomic Bulletin \& Review, 13, 402-408, ISSN 1069-9384

Towse, J. N. (1998). On random generation and the central executive of working memory. British Journal of Psychology, 89, 77-101, ISSN 2044-8295

Towse, J. N., \& Neil, D. (1998). Analyzing human random generation behavior: A review of methods used and a computer program for describing performance. Behavior Research Methods, Instruments $\mathcal{E}$ Computers, 30, 583-591, ISSN 0743-3808

Tippett, L. J., Waldvogel, H. J., Thomas, S. J., Hogg, V. M., van Roon-Mom, W., Synek, B. J., Graybiel, A. M., \& Faull, R. L. M. (2007). Striosomes and mood dysfunction in Huntington's disease. Brain, 130, 206-221, ISSN 0006-8950

Walker, F. O. (2007). Huntington's disease. Lancet, 369, 218-228, ISSN 0140-6736

Weir, D. W., Sturrock, A., \& Leavitt, B. R. (2011). Development of biomarkers for Huntington's disease. Lancet, 10, 573-590, ISSN 0140-6736

White, N. M. (1997). Mnemonic functions of the basal ganglia. Current Biology, 7, 164-169, ISSN 0960-9822

Yeung, N., \& Cohen, J. D. (2006).The impact of cognitive deficits on conflict monitoring: Predictable dissociations between the ERN and N2. Psychological Science, 17, 164171, ISSN 1467-9280

Zuccato, C., \& Cattaneo, E. (2007). Role of brain-derived neurotrophic factor in Huntington's disease. Progress in Neurobiology, 81, 294-330, ISSN 0301-0082

Zuccato, C., Valenza, M., \& Cattaneo, E. (2010). Molecular mechanisms and potential therapeutical targets in Huntington's disease. Physiological Reviews, 90, 905-981, ISSN 0031-9333 


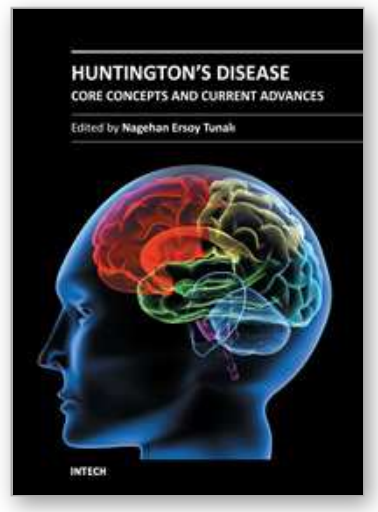

\author{
Huntington's Disease - Core Concepts and Current Advances \\ Edited by Dr Nagehan Ersoy Tunali
}

ISBN 978-953-307-953-0

Hard cover, 554 pages

Publisher InTech

Published online 15, February, 2012

Published in print edition February, 2012

Huntington's Disease is one of the well-studied neurodegenerative conditions, a quite devastating and currently incurable one. It is a brain disorder that causes certain types of neurons to become damaged, causing various parts of the brain to deteriorate and lose their function. This results in uncontrolled movements, loss of intellectual capabilities and behavioural disturbances. Since the identification of the causative mutation, there have been many significant developments in understanding the cellular and molecular perturbations. This book, "Huntington's Disease - Core Concepts and Current Advances", was prepared to serve as a source of up-to-date information on a wide range of issues involved in Huntington's Disease. It will help the clinicians, health care providers, researchers, graduate students and life science readers to increase their understanding of the clinical correlates, genetic aspects, neuropathological findings, cellular and molecular events and potential therapeutic interventions involved in HD. The book not only serves reviewed fundamental information on the disease but also presents original research in several disciplines, which collectively provide comprehensive description of the key issues in the area.

\title{
How to reference
}

In order to correctly reference this scholarly work, feel free to copy and paste the following:

Eddy J. Davelaar (2012). Computational Investigations of Cognitive Impairment in Huntington's Disease, Huntington's Disease - Core Concepts and Current Advances, Dr Nagehan Ersoy Tunali (Ed.), ISBN: 978-953307-953-0, InTech, Available from: http://www.intechopen.com/books/huntington-s-disease-core-conceptsand-current-advances/computational-investigations-of-cognitive-impairment-in-huntington-s-disease

\section{INTECH}

open science | open minds

\section{InTech Europe}

University Campus STeP Ri

Slavka Krautzeka 83/A

51000 Rijeka, Croatia

Phone: +385 (51) 770447

Fax: +385 (51) 686166

www.intechopen.com

\section{InTech China}

Unit 405, Office Block, Hotel Equatorial Shanghai

No.65, Yan An Road (West), Shanghai, 200040, China 中国上海市延安西路65号上海国际贵都大饭店办公楼 405 单元

Phone: +86-21-62489820

Fax: $+86-21-62489821$ 
(C) 2012 The Author(s). Licensee IntechOpen. This is an open access article distributed under the terms of the Creative Commons Attribution 3.0 License, which permits unrestricted use, distribution, and reproduction in any medium, provided the original work is properly cited. 